\title{
Emerging new practices in technology to support independent community access for people with intellectual and cognitive disabilities
}

\author{
Steven E. Stock ${ }^{\mathrm{a}, *}$, Daniel K. Davies ${ }^{\mathrm{a}}$, Michael L. Wehmeyer ${ }^{\mathrm{b}}$ and Yves Lachapelle ${ }^{\mathrm{c}}$ \\ a AbleLink Technologies, Inc., Colorado Springs, CO, USA \\ ${ }^{\mathrm{b}}$ University of Kansas, Lawrence, KS, USA \\ ${ }^{\mathrm{c}}$ University of Québec at Trois-Rivières, Québec, Canada
}

\begin{abstract}
The concept of community access is a multidimensional term, which may involve issues related to physical access, knowledge and information, power and control, relationships and communications, advocacy, participation and quality of life [21]. This paper discusses historical and emerging practices and interventions related to physical access to community and community based information for individuals with cognitive disabilities such as intellectual disability, autism or traumatic brain injury. While much societal attention has been paid to features of independent community access for populations such as individuals with hearing, vision or physical disabilities, less attention has focused on independent community access for people with intellectual and other significant cognitive disabilities. Attitudes and actions by families and professional service communities are often mixed for some individuals in this population. The somewhat limited research base in these areas is explored, including a case study review and results from several promising feasibility studies. The paper concludes with comments concerning future prospects and recommendations for improving independent community access for persons with significant cognitive disabilities.
\end{abstract}

Keywords: Intellectual and cognitive disabilities, technology, community access

\section{Introduction}

Independent access to community settings and the many benefits that can be realized therein are overwhelmingly accepted as positive aspects of mainstream society. Many if not most of our social, recreational, vocational, educational, consumer, business and health needs are met in community settings. Despite the availability of services through web sites and other internet resources, most of us still shop, attend medical appointments, go bowling, visit libraries, meet with friends,

*Corresponding author: Steven E. Stock, 618 N. Nevada Ave., Colorado Springs, CO 80903, USA. Tel.: +1 719592 0347; Fax: +1 719592 0248; E-mail: steve@ablelinktech.com. take classes, make bank transactions and work in community settings.

There are, however, problems in ensuring independent community access for people with intellectual and other significant cognitive disabilities. As institutions in America began closing in the early 1970s and as the number of community-based support services increased through the 1970 s and 80 s $[13,17]$ issues relating to individual safety and security also emerged as barriers to full inclusion in the community [2,14,20]. When we leave the safety of our homes we are naturally exposed to the greater risk that the uncertainty of community presence can present. We mitigate this greater exposure to risk and victimization in many ways; we lock our car doors, travel with others or at certain times of day, visually survey environments before entering, 
restrict travel to certain areas, and sometimes even carry defensive tools such as pepper spray or firearms.

For people with intellectual disability, certain learning disabilities, autism, and significant traumatic brain injuries, the give and take between the benefits and risks of independent community access must be continually weighed. However, while few would disagree that we have made significant progress in facilitating the safety and security needs of people with significant cognitive disabilities, society continues to struggle with mitigating fears in support of providing opportunities for increased community presence and the realization of benefits associated with independent access to the community.

This article provides a review of several barriers to independent community access for people with cognitive disabilities, as well as opportunities that technology may offer to overcome these barriers in the areas of travel, navigation and access to community based information. No technology, however, can completely assure community safety and therefore the narrative includes limitations of these emerging technologies as well as recommendations for future research that are necessary to better balance the issues of safety/security and improved quality of life.

\section{Community access for people with intellectual and cognitive disabilities}

While the impetus for providing greater community integration to and for people with intellectual and cognitive disabilities has been ongoing for several decades, there is surprisingly little empirical research on the topic. Indeed, in a systematic review of the literature between the years 1996 and 2006, Verdonschot and colleagues concluded that "... on the basis of empirical evidence, within the time frame of this literature search, little is known about community participation of people with ID. Many researchers did not clearly define community participation and were concerned with limited areas of community participation; research is seldom based on a theoretical framework" [26]. Nevertheless, we summarize the extant literature as we discuss particular technology supports.

\subsection{Personal navigation - Vehicle and walking}

Transportation issues have long been recognized as a primary barrier to independent community access for people with intellectual and cognitive disabilities [10,
18,27]. Due to personal capacity and financial factors, people with intellectual and other cognitive disabilities are much less likely to obtain a driver's license or own a personal vehicle than the general public. Other mainstream transportation options, such as taxi cabs, can also present financial barriers. Instead, a common mode of transportation used involves some type of agency or other specialized transportation [21]. This type of transportation provides high levels of safety and security, but also has significant limitations. For example, agency sponsored transportation may provide door-todoor pick up and drop off services, but may be limited in terms of hours of availability, travel purposes and destinations, and usually requires scheduling in advance. Further, these transportation services are generally segregated as they are only offered to clients of the agency's other services, and this is not philosophically aligned with notions of community integration.

Walking as a form of transportation also provides opportunity as well as drawbacks. A short walking route to a friend's home, the local convenience store or to a place of employment has historically been a form of independent access to community resources, but is obviously limited in its usefulness by distance. Other limitations to walking may include safety issues regarding encounters with strangers, getting lost, lack of accessible routes for persons with physical challenges, or busy street crossings. These limitations may be somewhat mitigated by the ubiquitous availability of cell phones, which are increasingly serving as a life line not only for people with disabilities traveling in the community [4], but also for mainstream populations such as younger children or older adults. Examples of cell phones with easy-to-use features include the Jitterbug (www.jitterbug.com/), the Firefly designed primarily for younger users (www. fireflymobile.com/), the Pocket ACE multimedia cell phone (www.ablelinktech.com/_handhelds/pocketace. asp), or the Doro PhoneEasy (www.consumercellular. com/Info/Phones\#Doros). It is important, however, that these units are properly programmed and users are subsequently trained to proficiency on their use prior to being used as an aid to community access.

\subsection{Public transportation}

Public transportation, often in the form of bus, train, subway, and even ferry systems, is an important resource for people who do not or cannot drive personal automobiles. The public benefit of city transit systems has long been accepted and is reflected in their long 
history of government subsidies. While public transportation options are likely to be more limited in rural areas, they can be a considerable asset to people with intellectual and cognitive disabilities in communities where such options exist. For people who cannot use mainstream public transportation, some governments include requirements for the provision of alternative services. For example, in the United States federal law [1] requires "complementary paratransit service, comparable to public fixed route systems." These types of transportation services are similar to agency programs in that they provide door-to-door pick up and drop off and do not operate on fixed routes or schedules. They also include some of the same limitations, such as requirements for advanced notices and limited operating times. In the United Kingdom where community transport services are provided by local councils, provisions for disability access to public transportation vary, ranging from door to door transport [9] to free off-peak travel on local bus services [10],

Mainstream public fixed-route transit systems provide potential for increasing community access and participation for people with significant cognitive impairments. Despite limitations in pickup/destination sites and schedules, public transportation enables many people to access work, social, educational and medical community resources. For people with intellectual and cognitive disabilities, there are challenges, however, to the independent use of public transit systems, ranging from the need to understand complex schedules to difficulties with transfers. Access to public transit for this population is also hampered by safety concerns on the part of family members and caregivers. The two most commonly cited reasons for limiting independent access to mainstream public transportation involve the potential of being victimized and fears of getting lost [12, $21,25]$.

Traditionally, various approaches involving handson training on transportation skills and communitybased instruction using specific bus routes have been implemented to promote access to transportation for people with intellectual and cognitive disabilities [2,3]. Recent evidence has emerged, however, on the potential for "computer based video instruction (CBVI)" for supporting independent community access, including teaching bus use [19]. This study involved creation of "a lifelike public bus riding scenario in a simulated environment to teach bus transportation skills" to three adolescents with intellectual disability. Video recordings were made from a first-person perspective and included voice over prompts such as "Look for Advanced
Auto Parts" and "Push the request for stop signal when you see the Chick-Fil-A and Target sign." Subsequent CBVI sessions were conducted in a classroom and provided via Microsoft PowerPoint software. A multiple probe design across participants and one bus route was used to evaluate the effectiveness of the CBVI approach to teach bus transportation skills. Results indicated that the CBVI approach was "an effective and efficient method for teaching students to locate landmarks and the target bus stop," as two of the three participants met criteria for correctly pushing the request to stop signal within the minimum possible number of five sessions. Additionally, during actual in-vivo sessions on the bus, two participants were able to generalize the skill with $100 \%$ correct performance. The third participant failed to push the signal only during the first in-vivo session due to requesting reassurance, and verbally acknowledged that "I should have pushed it" after passing the stop. She subsequently completed the task correctly on the remaining two trials. In terms of skill maintenance, the three participants were able to demonstrate the ability to correctly push the request to stop signal ranging from 7 to 52 days.

The use of Global Positioning System (GPS) technology to support transportation and navigation has become commonplace and offers the potential for supporting the mobility needs of people with disabilities. Mainstream GPS navigation devices provide numerous features such as detailed on-screen maps and graphic lane guidance, traffic data, point of interest information, text-to-speech directions, complete cell phone functionality, web browsing and other information functions, and speech input operation. While these devices are likely used primarily for vehicle navigation, they can also be used to obtain directions while walking. Individuals with intellectual disabilities may benefit from the latter use, given that this population is much more unlikely to drive a personal vehicle and the technology is limited in its support of travel on public transit systems (i.e., landmark information may be helpful but turn-by-turn directions are not useful to passengers). Other persons with cognitive disability symptoms that do not sufficiently impair their ability to drive (such as in some mild brain injuries) may benefit from mainstream GPS technologies, but these users may also be overwhelmed by the relative complexity and robust feature set of these devices. As with other mobile devices, the distractive nature of GPS units may potentially endanger drivers, and both individual evaluation and more long range research should be conducted on their efficacy for cognitively impaired populations. 
Other recent developments concerning GPS technology are also worth noting. For example, the company HumanWare distributes several GPS based navigation products designed specifically for people who are blind or who have severe low vision [15]. Several initial investigations have occurred relating to the potential of GPS technology to benefit independent mobility for students and adults with intellectual disability. In one project, researchers at the University of Colorado's Cognitive Levers project engaged in a project called Mobility for All (MfA), which developed a hand held prototype system that worked with the on board GPS technology used by the local public transit system. The MfA prototype provided visual and auditory cues to users similar to those provided in one-on-one training programs to support "critical tasks including which bus to board, when to get off, and where to go next" [24]. And while the prototype included features for trip monitoring and error detection, its dependency on cooperation from public transit providers limited commercialization opportunities for the system.

A series of studies have been conducted by the authors that involved development and evaluation of a GPS software application designed to support independent use of public transportation by students and adults with intellectual disabilities. The first of these [7] provided evidence that the approach of using GPS technology to enable authoring of multimedia instructions for navigating specific municipal bus routes could reduce travel training requirements for this population. Researchers used the prototype system's configuration utility while traveling the designated route, setting GPS waypoints and recording corresponding instructional cues that were automatically launched based upon GPS location during route playback by study participants. Examples of audio cues included "this is not your stop, please do not get off the bus here" or "you will be getting off at the next bus stop, so pull the cord now to ring the bell." Digital picture cues were also provided at designated GPS waypoints for landmarks along the route, which were also supported by audio cues such as "you are now going past the post office, so you are about half way to the bowling alley." Figures 1-3 provide samples of the software system's interface for route creation and playback.

This study provided initial evidence of the efficacy of the customized GPS approach by demonstrating a reduction in bus training time required to learn a new route. In a follow up study [5], 26 participants with intellectual disabilities used the software prototype operating on a GPS enabled personal digital assistant to attempt independent navigation of a bus route, without being accompanied by a researcher or other travel companion on the bus and without prior knowledge of the intended destination.

In the follow up study, participants first learned how to use the system by traveling on a training route with a trainer, followed by traveling another route using the system but without the accompaniment of a trainer. Finally, participants used the system to travel a route without accompaniment and without knowledge of the destination. During this trial, 24 of the 26 participants exited the bus at the correct location. Along with the postive implications for increasing independent community access opportunities for people with intellectual disability, these studies also highlighted potential benefits in the area of reduced training costs. For example, in the United States many individuals with intellectual disabilities who are not able to use standard city buses currently use paratransit services. Data provided by a mid sized US urban city transit system documented that the cost savings of being able have a rider with a disability learn to use the regular bus, rather than using paratransit, is $\$ 4,511.52$ per year. This is due to the high cost of paratransit service as compared to standard bus service. Thus the prototype system's potential for training riders with fewer trials can provide significant cost savings to the local government or other transortation training entities. The study finding in which 24 of $26(92 \%)$ of test participants were able to successfully exit the bus without prior knowledge of the target destination was particulary striking. Several participant comments recorded after study sessions are provided below. In summary, use of the multimedia GPS-based software approach to increase independent access to the community for indivdiuals with significant cogntive challenges has significant potential and is worthy of further investigation.
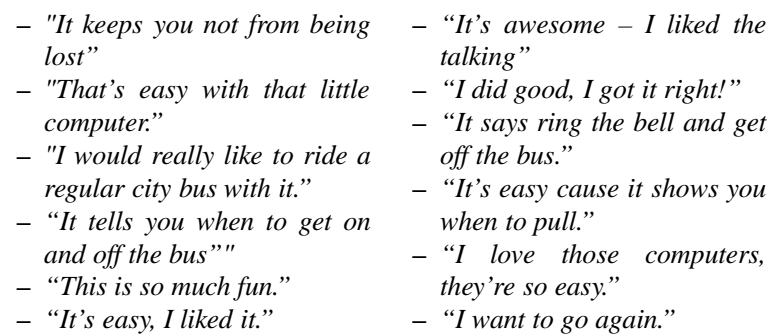

Another technology that could be a positive asset in promoting opportunities for community access involves the industry of tracking devices. These technologies, such as the Brickhouse Child Locator or 

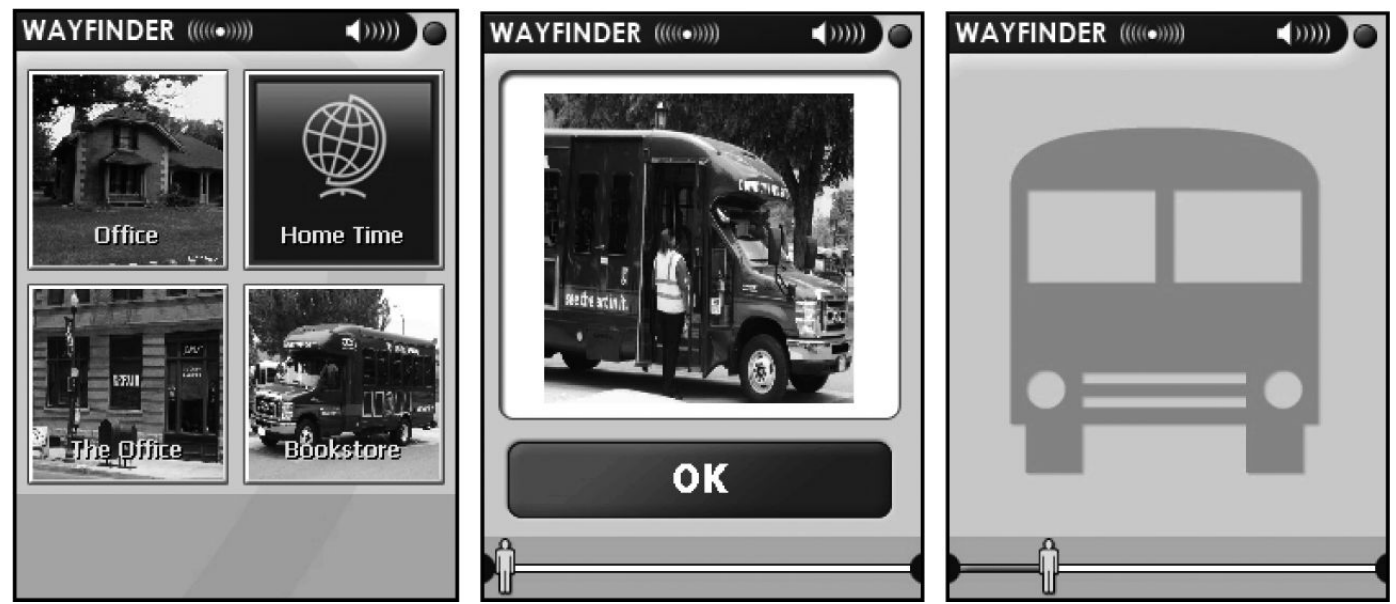

Fig. 1. WayFinder's Main Menu showing four travel routes (left); at beginning of route (center) and during transit (right). Note progress bar showing relative route position at bottom of screen.
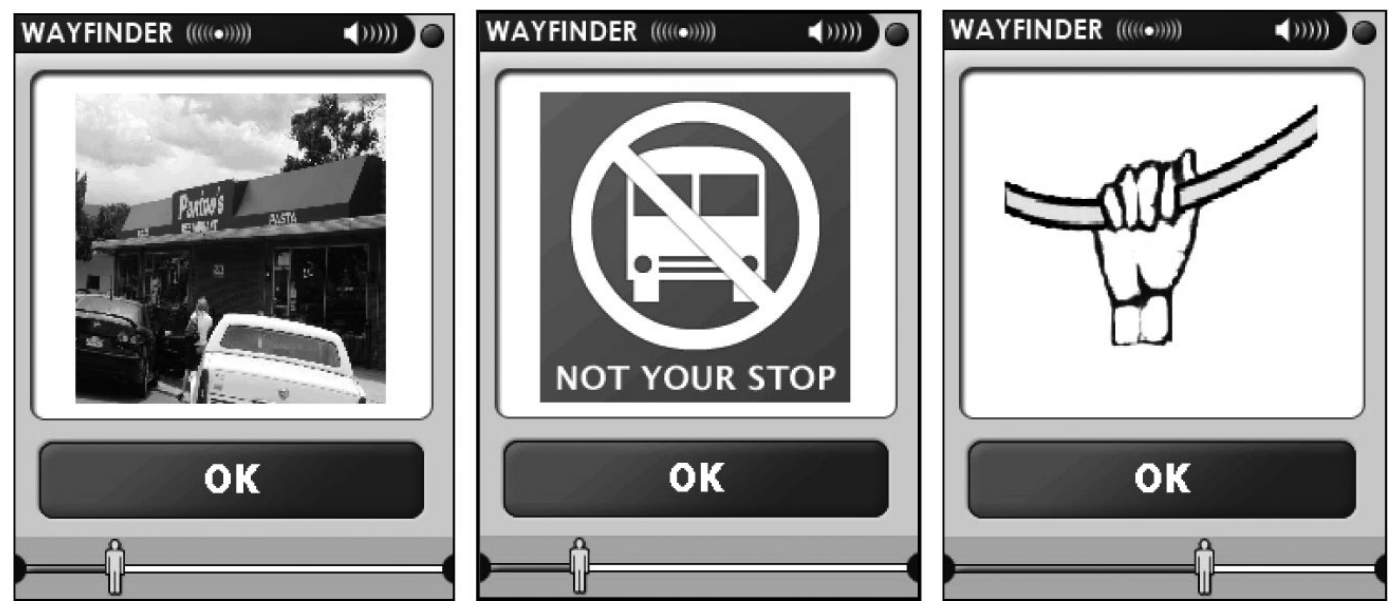

Fig. 2. WayFinder display showing a Landmark waypoint (left); a Not-Your-Stop waypoint (center), and a Signal Stop waypoint (right).
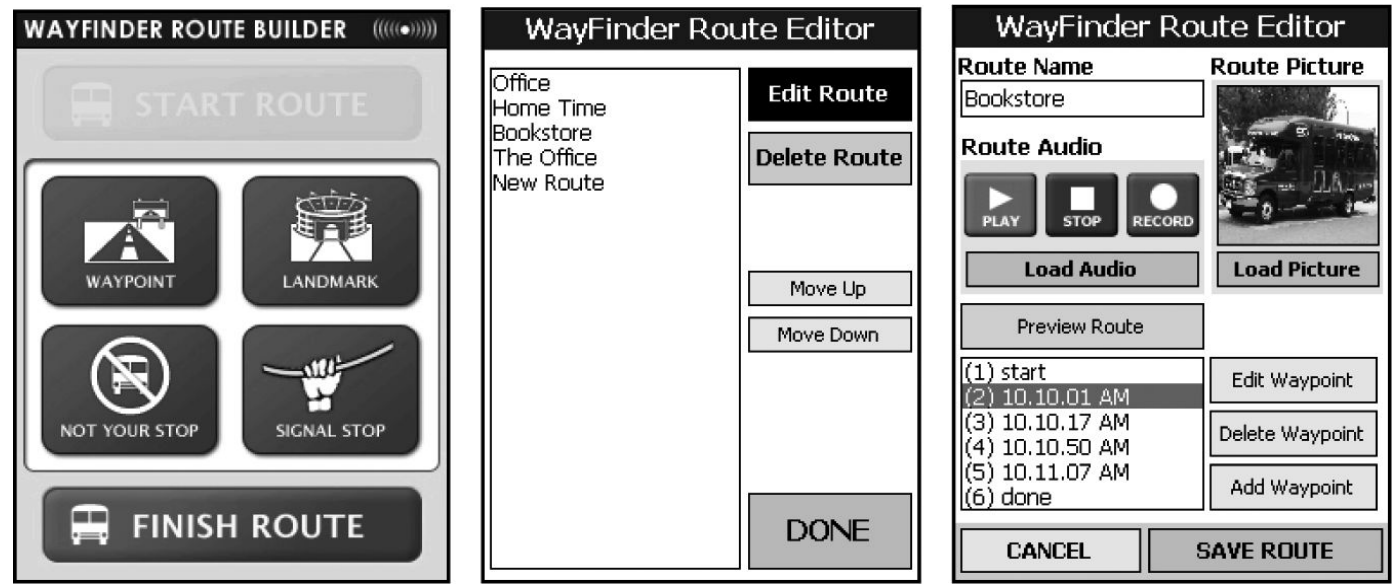

Fig. 3. Route Builder interface with waypoint options (left) and Route Editor interface (center and right). 
Loc8tor Plus, generally require the individual being tracked to wear a device such as a wristband or pendant that emits a signal to a controller unit. Controller units then emit a warning alarm when the signal emitting device moves beyond a designated range. These types of tracking devices are designed more for individuals with wandering behaviors and can be limited in tracking range. Other systems may be more suitable for individuals with cognitive limitations who are purposefully traveling in the community, such as the Spark Nano Tracker, Livewire FastTrak, Community Sidekick or KoolTrax systems. These devices are often similar in size and form to a cell phone (or as a software download to an existing cell phone), and can provide automated location information as a link to a web based map to one or more designated email addresses or cell phones. The individual authorized to monitor the user's location then activates the link on the web enabled phone or in the email message to open a browser page displaying the user's location within a general range of 5 to 10 meters (or 16 to 33 feet). This type of technology does not have limits regarding distance, but may require a monthly or annual subscription fee along with the initial product purchase price.

\subsection{Navigation within buildings}

While GPS technology and multimedia software interfaces have been shown to provide potential for increasing opportunities for independent community access, the technology can lose its effectiveness once a traveler enters building facilities where GPS signals become limited or non existent. Similar to travel training, repetitious, one-on-one on-site training is generally used to assist persons with cognitive disabilities to learn to navigate to desired spaces inside of buildings such as doctor's offices or shopping malls. Research has shown that highlighting landmarks along navigation routes in indoor settings can be a useful strategy during travel repetition priming [9, 22]. More current supports are being investigated, although most available research has focused on independent indoor navigation by individuals with vision disabilities. For example, there may be potential for the use of computer based video prompting similar to the previously cited research of Mechling and O'Brien [19] to support independent indoor navigation [16], and this should be explored in future research endeavors. Currently there are several step-bystep multimedia prompting devices available that could be suited for this purpose. These range from the sim- ple and affordable audio-based Step Pad by Attainment Co. (www.attainmentcompany.com/), to the full multimedia, smart phone based Visual Assistant system by AbleLink Technologies (www.ablelinktech.com). The case study in section 4 of this manuscript describes just such an implementation.

\subsection{Access to community information}

Along with transportation, a second area of importance in accessing the benefits of community settings is access to information sources, such as signs, kiosks, building directories, exhibit displays, and other information. For people with intellectual disability, the fact that these types of information are most often in text format presents a barrier to greater access. For example, many zoos or walking tours provide either stationary signage with text or a written guide to follow to access relevant information. This area has received even less attention by the research community than transportation issues.

The increasing frequency of touch screen, multimedia kiosk-based information represents an example of how universally designed information sources often benefits everyone. Another means of accessing community information may be in using the internet on a screen-reader enabled computer to research desired subjects prior to community travel, but this is obviously limited and less than ideal. More recently, large amusement parks, zoos and other facilities have begun developing electronic tour guides that use pictures, audio and video to provide information about various points of interest in their fixed environments. This form of information access generally runs on portable devices such as personal digital assistants and is activated via wireless signals such as in Bluetooth, triangulation, infrared or (if outdoors) GPS based signals. The benefit of these systems was demonstrated in a research and development project conducted by the authors [6] that showed significant advantages over traditional paperbased tour information in terms of accessing information from a historic walking tour, where 23 participants with intellectual disabilities were able to use a GPSbased system with significantly more independence and with fewer errors than when using the tour's brochure information.

Given this background on various issues regarding independent community access, the following case study is provided to provide a real life illustration of the potential benefit that some of these technologies may provide. 


\section{Joel: A case study}

This research was conducted by a Canadian university with the general goals of (a) translating into French and adapting two community access software systems operating on a hand held computer, (b) field-testing these applications by assessing their utility to help a person travel independently to and from four different locations, and (c) determining to what extent these technologies could help a person in decision-making and problem solving. The pilot study involved a 19 year old young man with Down Syndrome. The main goal was to use the technologies as supports to enable safe travel back and forth from four different locations within the city of Montreal, Canada. For this study, two such software applications developed by Able Link Technologies were used. The first, Discovery Desktop, replaces the Windows Mobile interface for a simplified interface that allows the person to run customized applications represented by large icons. The second, Visual Assistant, is an application to combine photographs or video segments with verbal instructions for step-bystep prompting.

The research participant (Joel) was provided a Smartphone equipped with the two applications to help him complete his travels. Four new travel routes were identified to be used for the study. These destinations were chosen according to the Joel's personal interests. They involved going to his sister's apartment, the national library and two retail shops. Joel did not have previous experience traveling these routes using public transportation. For each trip, a step-by-step travel script was developed, representative photographs were taken and the multimedia instructions were configured and uploaded for use in the Visual Assistant system. To provide additional reassurance for Joel's parents, an HTC 6800 Smartphone was used to allow him, if necessary, to call home for assistance.

Before beginning use of the device, a 30 minute semistructured pre test interview was conducted with Joel's parents. This interview included documenting Joel's background information, desired travel routes, and the parents' perception in regard to the proposed technology. An interview with Joel was also completed in order to evaluate his self-determination skills. Two instruments were used to make these assessments. These included a written interview outline along with the Adolescent-Young Adults (16-21 years) French version of The Arc's Self-Determination Scale [28]. These evaluations indicated that Joel's sense of self-determination was relatively high on all dimensions except for Self-
Regulation, which was in the lower 60th percentile. Still, compared to peers with intellectual disabilities he was somewhat more self-determined than average.

Following these preliminary activities, the evaluation period began. Joel was asked to choose his desired sequence of travels. Except for some minor challenges like environmental noise within the subway car, the sun reflection on the screen and one instance of making a right turn instead a left (corrected by successfully asking for assistance), the results were extremely positive. Joel completed two trips at first with assistance before he expressed confidence in traveling alone without needing a support person. All subsequent trips where then made by Joel independently.

Following completion of the sequence of travel trips, post-test assessments using the interview instrument were again completed with Joel's parents. This time they were asked about their feelings on Joel traveling with the help of the technology and their perceptions in regard to the benefit of the technology used. Joel was again evaluated with The Arc's Self-Determination Scale and was also asked to complete the Quebec User Evaluation of Satisfaction with Assistive Technology (QUEST 2.0) [8]. The first part of this questionnaire consists of eight items asking a person to rate his level of satisfaction on a 5-point Likert-type scale on the technology being used, including ratings on dimension, weight, ease of use, and efficacy. The second part asks the respondent to rank the most important system components on a scale from 1 to 3 .

Although it appeared that the use of the technology for the duration of the case study did not affect Joel's self-determination since the pre/post tests score at the Self-Determination scale were almost the same, researchers believe that the scale's effectiveness was likely limited due to the short 1-2 month period of the technology intervention. Despite these findings, comments from the parents, the travel trainer and Joel were all highly positive and indicated their personal perceptions that the technologies provided an important impact on Joel's choice-making, decision-making and problem solving. In terms of Joel's satisfaction with the technology, he scored all eight satisfaction items on the Likert scale as a 4 or 5, (i.e., satisfying or very satisfying). Joel also ranked dimension, ease of use and efficacy as the top three components of the system.

Qualitative analyses of the parent and trainer pre/post interviews were done using N'Vivo. Main results showed that Joel was happy, excited and confident. Joel's mother indicated she was pleased with the size of the technology but was a bit anxious about the safe- 
ty of her son when using it. However, she was positively impressed when her son once went to a stranger in the subway to ask for help. Joel's father reported that after the first use (to get to his sister's residence) Joel had told him "It's easy to use... I love it and it went just fine." (Note: Quotes herein were provided in French and translated by research staff supporting the project.) The second use was to get to the local library. Once back home Joel announced to his parents that he "could now do it alone... with the technology." His father stated "I am convinced of the effectiveness of this assistant for independent travel. I believe it can significantly promote self-determination for people with disabilities. Also, it can help increase self-esteem for the person who finds he or she can quickly and easily perform a task without assistance or supervision of a teacher or parent. Thanks to this device, Joel is able to travel by himself!" The trainer further observed that Joel was "excited, enthusiastic and effective at using the technology right from the beginning...just like he had played with the technology before..."

Researchers summarized that the most important outcome of the study was the technology was able to assist Joel in using public transportation and making his community more accessible. They further concluded that although chosen tasks for this research concerned travels, further research on this type of software should be conducted to evaluate its impact on helping people perform other community-based activities of daily life.

\section{Conclusions and recommendations}

The debate for increasing independent community access opportunities and options for people with significant cognitive disabilities has, and will continue, to be centered on the dignity of risk vs. erring on the side of safety. As was shown in Joel's case study, technology that has been subject to hands-on research can provide effective support to increase opportunities in this area. While other work cited in this article has demonstrated the potential of new technologies to support independent community access, much more research is needed to determine the types and distance of travel that can be effectively supported, experiments on familiar vs. unfamiliar routes, transfers, the nature of populations that can benefit from its use, and other important benefits and limitations of these general approaches. However, no amount of clinical research may supplant the need for individualized trials - such as Joel's - to assuage the concerns of parents and other caregivers in terms of safety and individual efficacy. These initial individualized trials should always include backup strategies (such as one-to-one support and observation during initial trials, and use of tracking technologies where appropriately authorized). Additionally, it is critical to ensure that candidates for using way finding technologies such as those discussed in this article have adequate skills - such as self rescue skills and knowledge of appropriate community interaction - before being matched with a technology solution. Thus, initial research into the use of transportation and information access technologies for people with cognitive disabilities has provided promising results and indicates the need for continued research and development of cognitively accessible technologies to facilitate community access. This may include usability studies of existing technologies by different populations characterized by cognitive disabilities, efficacy studies to determine impacts and limits in areas such as frequency of community access, quality of life and community safety, and research and development of new technology approaches and solutions.

\section{References}

[1] A.D.A. Portal, The ADA Transportation Series, obtained from web site at http://adaportal.mtc-inc.com/Transportation/ FAQ/City_Bus_Systems.html on July 22, 2010.

[2] M. Agran, Promoting Health and Safety, Skills for Independent Living (1994), 221.

[3] E. Bourland, Travel Training for Youth with Disabilities, NICHCY Transition Summary 9 (June 1996).

[4] D.N. Bryen, A. Carey and M. Friedman, Cell Phone Use by Adults with Intellectual Disabilities, Intellectual and Developmental Disabilities 45(1) (2007), 1-2.

[5] D.K. Davies and S.E. Stock, WayFinder: A Cognitive Aid for Independent Transportation, Phase I Final Progress Report submitted to the National Institutes on Health, 1 July 2008.

[6] D.K. Davies and S.E. Stock, Development and Evaluation of a Location Based Multimedia System for Providing Access to Information in Community Settings for Students and Adults with Intellectual Disabilities, Phase I Final Project Report submitted to the US Department of Education, 31 Mar 2009.

[7] D.K. Davies, S.E. Stock, S. Holloway and M.L. Wehmeyer, Evaluating a Cognitively Accessible GPS-Based Transportation Assistance PDA to Enable Independent Bus Travel for People with Intellectual Disability, Intellectual and Developmental Disability; accepted for publication, 2010.

[8] L. Demers, R. Weiss-Lambrou and B. Ska, The Quebec User Evaluation of Satisfaction with Assistive Technology (QUEST 2.0): An overview and recent progress, Technology and Disability 14 (2002), 101-105.

[9] Directgov, Community transport and Shopmobility, obtained from web site at: http://www.direct.gov.uk/en/Disabled People/MotoringAndTransport/PublicAndCommunityTran sport/DG_073262 on 15 December 2010. 
[10] Directgov, Community transport and Shopmobility, obtained from web site at: http://www.direct.gov.uk/en/Disabled People/MotoringAndTransport/PublicAndCommunityTran sport/DG_4019388 on 15 December 2010.

[11] P. Foo, W.H. Warren, A. Duchon and M.J. Tarr, Do Humans Integrate Routes Into a Cognitive Map? Map-Versus LandmarkBased Navigation of Novel Shortcuts, Journal of Experimental Psychology: Learning, Memory, and Cognition 3(2) (2005), 195-215.

[12] S.R. Glausier and J.E. Whorton, Leisure Put Into PerspectiveWill the Circle Go Unbroken? Catalyst 34(1) (2000), 11-19.

[13] M.W. Gold, An Adaptive Behavior Philosophy: Who Needs It? Research report published May (1972).

[14] A.S. Halpern, An Empirical Analysis of the Dimensions of Community Adjustment for Adults with Mental Retardation in Semi-Independent Living Programs, Australia and New Zealand Journal of Developmental Disabilities 12(3) (1986), 147-157.

[15] HumanWare, HumanWare Products, obtained from web site at: http://www.humanware.com/en-usa/products, on 22 July 2010.

[16] N.L. Kirsch, M. Shenton, E. Spirl, J. Rowan, R. Simpson, D. Schreckenghost and E.F. LoPresti, Web-Based Assistive Technology Interventions for Cognitive Impairments After Traumatic Brain Injury: A Selective Review and Two Case Studies, Rehabilitation Psychology 49(3) (2004), 200-212.

[17] R.B.. Kugel and W. Wolfensberger, Changing Patterns in Residential Services for the Mentally Retarded, A President's Committee on Mental Retardation Monograph, published 10 Jan 1969.

[18] M. Lee and D. Verban, Parents Alliance Employment Project: Moving Ahead with Integrated Employment, An Update, Third Edition, (ED331257), 1990.

[19] L. Mechling and E. O'Brien, Computer-Based Video Instruction to Teach Students with Intellectual Disabilities to Use Public Bus Transportation, Education and Training in Autism and Developmental Disabilities 45(2) (2010), 230-241.
[20] L. Nihira and K. Nihira, Jeopardy in Community Placement, American Journal of Mental Deficiency 79(5) (1975), 538544.

[21] M. Nind and J. Seale, Concepts of access for people with learning difficulties: towards a shared understanding, Disability and Society 24(3) (2009), 273-287.

[22] M.J. Reardon, D.G. Frazier and G. Tonks, Getting on Board: A Review of Transportation Options for Ohioans with Disabilities and Recommendations for Systems Change. Final Report to the Ohio Developmental Disabilities Council on Grant Project 98-4 Transportation, March 1993.

[23] B.J. Stankiewicz and A.A. Kalia, Acquisition of structural versus object landmark knowledge, Journal of Experimental Psychology: Human Perception and Performance 33(2) (2007), 378-390.

[24] J. Sullivan, Mobility-for-All: Community Access through Intelligent Mass Transportation Systems, project description obtained from web site at http://13d.cs.colorado.edu/clever/ assets/flyer/mfa.pdf on 22 June 2010.

[25] V.A. Temple, Factors Associated with High Levels of Physical Activity among Adults with Intellectual Disability, International Journal of Rehabilitation Research 32(1) (2009), 89-92.

[26] M.L. Verdonschot, L.P. de Witte, E. Reichrath, W.E. Buntinx and L.G. Curfs, Community Participation of People with an Intellectual Disability: A Review of Empirical Findings, Journal of Intellectual Disability Research 53(4) (2009), 303-318.

27] M.M.L. Verdonschot, L.P. de Witte, E. Reichrath, W.H.E. Buntinx and L.M.G. Curfs, Impact of Environmental Factors on Community Participation of Persons with an Intellectual Disability: A Systematic Review, Journal of Intellectual Disability 53(1) (2009), 54-64.

[28] M.-L. Wehmeyer, Y. Lachapelle, D. Boisvert, D. Leclerc and R. Morrissette, The Self Determination Scale for Adolescents, Laboratory of Interdepartmental Research in Intellectual Deficiency, University of Québec at Trois-Rivières, 2001. 